\title{
Bandwidth limitations in current mode and voltage mode integrated feedback amplifiers
}

\section{Bruun, Erik}

\section{Published in:}

Proceedings of the IEEE International Symposium on Circuits and Systems

Link to article, DOI:

10.1109/ISCAS.1995.521511

Publication date:

1995

Document Version

Publisher's PDF, also known as Version of record

Link back to DTU Orbit

Citation (APA):

Bruun, E. (1995). Bandwidth limitations in current mode and voltage mode integrated feedback amplifiers. In Proceedings of the IEEE International Symposium on Circuits and Systems (Vol. Volume 1, pp. 303-306). IEEE. https://doi.org/10.1109/ISCAS.1995.521511

\section{General rights}

Copyright and moral rights for the publications made accessible in the public portal are retained by the authors and/or other copyright owners and it is a condition of accessing publications that users recognise and abide by the legal requirements associated with these rights.

- Users may download and print one copy of any publication from the public portal for the purpose of private study or research.

- You may not further distribute the material or use it for any profit-making activity or commercial gain

- You may freely distribute the URL identifying the publication in the public portal 


\title{
Bandwidth Limitations in Current Mode and Voltage Mode Integrated Feedback Amplifiers
}

\author{
Erik Bruun \\ Electronics Institute, Bldg. 349 \\ Technical University of Denmark \\ DK-2800 Lyngby \\ Denmark
}

Tel.: +45 459342 07, Fax: +4545880117

E-mail: bruun@ei.dtu.dk

\begin{abstract}
The bandwidth relations of feedback amplifiers have been the subject of intense interest in recent years. Many current mode devices such as current feedback amplifiers have appeared which seem to break what has been considered almost a fundamental law that the product of closed loop gain and closed loop bandwidth remains constant for a feedback amplifier. The constant-bandwidth relations of such amplifler designs are reviewed in this paper and they are combined with the constraints imposed by technology when the feedback amplifier is to be designed in an integrated technology. From this analysis it is concluded that although very high performance designs are indeed feasible with the constant-bandwidth configurations, there are no constant-bandwidth configurations which provide a higher potential loop gain and closed loop bandwidth than the constant gainbandwidth product configurations.
\end{abstract}

\section{INTRODUCTION}

In recent years much attention has been paid to various forms of current mode operational amplifiers because of their gain independent bandwidth relations when used in feedback amplifier configurations, see e.g. [1]. An analysis of the bandwidth properties of all four basic opamp types (voltage opamp, current opamp, transresistance opamp, transconductance opamp) was recently presented by Toumazou, Payne and Lidgey in [2]. In this analysis it is shown that the utilization of the constant bandwidth properties relies on the possibility of isolating the opamp from the input source impedance and the output load impedance by the use of ideal current or voltage unity-gain buffers. However, as pointed out in [2] it is not a trivial task to design an ideal buffer. In practice, the input and output impedances are limited by technology constraints related to the maximum value of device transconductance

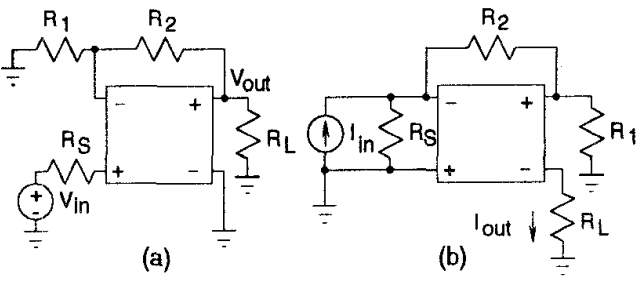

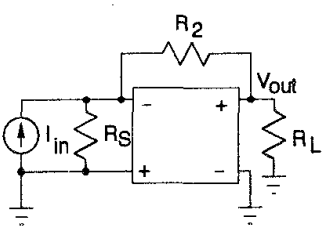

(c)

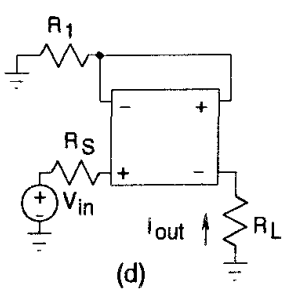

(d)
Fig. 1. Feedback amplifier configurations, [2]. (a) V-V converter. (b) I-I converter. (c) I-V converter. (d) V-I converter.

$g_{m}$ and the minimum value of device parasitic capacitance $C_{T}$ achievable from the technology applied.

In this work we take the analysis a step further and review the bandwidth properties assuming some generally applicable technology constraints concerning the achievable impedance levels.

\section{Feedback Amplifier Configurations}

Basically, there are four types of feedback amplifiers to consider: Voltage to voltage converter, current to current converter, current to voltage converter, and voltage to current converter. The feedback configurations for each of these amplifiers are shown in Fig. 1. Ideally, the opamp used for the feedback amplifiers in Fig. 1 is a nullor, i.e. a two-port where the input port is characterized by a differential input voltage of zero and an input current of zero, 


\begin{tabular}{lcccc}
\hline & $\begin{array}{c}\mathrm{V}-\mathrm{V} \\
\text { converter }\end{array}$ & $\begin{array}{c}\mathrm{I}-\mathrm{I} \\
\text { converter }\end{array}$ & $\begin{array}{c}\mathrm{I}-\mathrm{V} \\
\text { converter }\end{array}$ & $\begin{array}{c}\mathrm{V}-\mathrm{I} \\
\text { converter }\end{array}$ \\
\hline $\begin{array}{l}\text { Closed } \\
\text { loop } \\
\text { gain, } G_{0}\end{array}$ & $1+\frac{R_{2}}{R_{1}}$ & $1+\frac{R_{2}}{R_{1}}$ & $R_{2}$ & $\frac{1}{R_{1}}$ \\
\hline
\end{tabular}

Table 1. Ideal closed loop gain $G_{0}$ of feedback amplifier configurations.

and the output port has an arbitrary current-voltage relationship. With this ideal opamp the closed loop gain $G_{0}$ given in Table 1 results for each of the feedback amplifiers. In practice, the opamp is one of the following four types: Voltage opamp (voltage controlled voltage source, VCVS), current opamp (current controlled current source, CCCS), transresistance opamp (current controlled voltage source, CCVS), or transconductance opamp (voltage controlled current source, VCCS). The opamp will have a limited bandwidth, and assuming that it is characterized by a single dominant pole, the gain of the opamp can be expressed as

$$
A(s)=A_{0} \frac{1}{1+s / \omega_{0}}
$$

where $\omega_{0}$ is the angular frequency of the dominant pole and $A_{0}$ is the low frequency gain. Depending on the type of opamp, $A_{0}$ is a voltage gain $\left(A_{v}\right)$, a current gain $\left(A_{i}\right)$, a transresistance $\left(R_{t}\right)$, or a transconductance $\left(G_{t}\right)$.

From (1) we see that the gain-bandwidth product $G B$ of the opamp is $2 \pi G B=A_{0} \omega_{0}$. Note that the gainbandwidth product has the dimension of frequency for the voltage opamp and the current opamp only. For the transresistance opamp it has the dimension of the reciprocal of a capacitance, and for the transconductance opamp it has the dimension of the reciprocal of an inductance. Using (1) when analyzing the amplifier configurations in Fig. 1 the loop gain of each of the feedback amplifier configurations can be calculated for each of the four opamp types. The results of this calculation are shown in Table 2 which gives the low frequency loop gain for all 16 possible combinations of feedback amplifier configurations and opamp types. In all 16 combinations the loop gain has a single dominant pole at the angular frequency $\omega_{0}$. Note that the loop gain is dimensionless in all 16 cases. The loop gain is a most important parameter in a feedback system, partly because it determines the bandwidth, and partly because it reflects the improvements in distortion properties, impedance levels, component sensitivities, etc., which is obtained by the feedback. The closed loop bandwidth can be found as the frequency where the magnitude of the loop gain is reduced to one. With this simple interpretation of the loop gain we find that the closed loop bandwidth is simply $\omega_{0} /(2 \pi)$ multiplied by the low frequency loop gain listed in Table 2. Introducing the opamp $G B=A_{0} \omega_{0} /(2 \pi)$ and the closed loop low frequency gain $G_{0}$ we may compile the list of closed loop bandwidth expressions given in Table 3 .

From Table 3 it is also apparent that if the source resistance $R_{S}$ and/or the load resistance $R_{L}$ can be set to zero or infinity, the closed loop gain $G_{0}$ will drop out of all the bandwidth expressions, except for the expressions in the diagonal from upper left to lower right. This indicates that with source and/or load isolation most of the feedback amplifiers in Fig. 1 has a closed loop bandwidth which is not directly related to the closed loop gain. This is the result achieved in [2].

\begin{tabular}{|c|c|c|c|c|}
\hline $\begin{array}{l}\text { Opamp } \\
\text { type }\end{array}$ & $\begin{array}{c}\mathrm{V}-\mathrm{V} \\
\text { converter }\end{array}$ & $\begin{array}{c}\mathrm{I}-\mathrm{I} \\
\text { converter }\end{array}$ & $\begin{array}{c}\text { I-V } \\
\text { converter }\end{array}$ & $\begin{array}{c}\mathrm{V}-\mathrm{I} \\
\text { converter }\end{array}$ \\
\hline VCVS & $\frac{A_{v}}{1+\frac{R_{2}}{R_{1}}}$ & $\frac{A_{v}}{1+\frac{R_{2}+R_{L}}{R_{S}}+\frac{R_{L}}{R_{1}}+\frac{R_{2} R_{L}}{R_{1} R_{S}}}$ & $\frac{A_{v}}{1+\frac{R_{2}}{R_{S}}}$ & $\frac{A_{v}}{1+\frac{R_{L}}{R_{1}}}$ \\
\hline CCCS & $\frac{A_{i}}{1+\frac{R_{2}+R_{S}}{R_{L}}+\frac{R_{S}}{R_{1}}+\frac{R_{2} R_{S}}{R_{1} R_{L}}}$ & $\frac{A_{i}}{1+\frac{R_{2}}{R_{1}}}$ & $\frac{A_{i}}{1+\frac{R_{2}}{R_{L}}}$ & $\frac{A_{i}}{1+\frac{R_{S}}{R_{1}}}$ \\
\hline CCVS & $\frac{R_{t}}{R_{2}+R_{S}+\frac{R_{2} R_{S}}{R_{1}}}$ & $\frac{R_{t}}{R_{2}+R_{L}+\frac{R_{2} R_{L}}{R_{1}}}$ & $\frac{R_{t}}{R_{2}}$ & $\frac{R_{t}}{R_{S}+R_{L}+\frac{R_{S} R_{L}}{R_{1}}}$ \\
\hline VCCS & $\frac{G_{t}}{\frac{1}{R_{1}}+\frac{1}{R_{L}}+\frac{R_{2}}{R_{1} R_{L}}}$ & $\frac{G_{t}}{\frac{1}{R_{1}}+\frac{1}{R_{S}}+\frac{R_{2}}{R_{1} R_{S}}}$ & $\frac{G_{t}}{\frac{1}{R_{S}}+\frac{1}{R_{L}}+\frac{R_{2}}{R_{S} R_{L}}}$ & $\frac{G_{t}}{1 / R_{1}}$ \\
\hline
\end{tabular}

Table 2. Loop gain of feedback amplifiers. 


\begin{tabular}{lcccc}
\hline $\begin{array}{l}\text { Opamp } \\
\text { type }\end{array}$ & $\begin{array}{c}\mathrm{V}-\mathrm{V} \\
\text { converter }\end{array}$ & $\begin{array}{c}\text { I-I } \\
\text { converter }\end{array}$ & $\begin{array}{c}\text { I-V } \\
\text { converter }\end{array}$ & $\begin{array}{c}\text { V-I } \\
\text { converter }\end{array}$ \\
\hline VCVS & $\frac{G B}{G_{0}}$ & $\frac{G B}{1+\frac{R_{2}}{R_{S}}+\frac{R_{L}}{R_{1}}+G_{0} \frac{R_{L}}{R_{S}}}$ & $\frac{G B}{1+\frac{G_{0}}{R_{S}}}$ & $\frac{G B}{1+G_{0} R_{L}}$ \\
\hline $\operatorname{CCCS}$ & $\frac{G B}{1+\frac{R_{2}}{R_{L}}+\frac{R_{S}}{R_{1}}+G_{0} \frac{R_{L}}{R_{S}}}$ & $\frac{G B}{G_{0}}$ & $\frac{G B}{1+\frac{G_{0}}{R_{L}}}$ & $\frac{G B}{1+G_{0} R_{S}}$ \\
\hline $\operatorname{CCVS}$ & $\frac{G B}{R_{2}+G_{0} R_{S}}$ & $\frac{G B}{R_{2}+G_{0} R_{L}}$ & $\frac{G B}{G_{0}}$ & $\frac{G B}{R_{S}+R_{L}+G_{0} R_{S} R_{L}}$ \\
\hline $\operatorname{VCCS}$ & $\frac{G B}{1 / R_{1}+G_{0} / R_{L}}$ & $\frac{G B}{1 / R_{1}+G_{0} / R_{S}}$ & $\frac{G B}{1 / R_{S}+1 / R_{L}+G_{0} /\left(R_{S} R_{L}\right)}$ & $\frac{G B}{G_{0}}$ \\
\hline
\end{tabular}

Table 3. Closed loop bandwidth of feedback amplifiers.

\section{Technology Constraints}

In practice, in integrated circuit design, the technology will not permit the design of ideal impedance isolation buffers. For a given technology (e.g. CMOS or bipolar) we may assume a maximum usable device transconductance on the order of $g_{m}$, a minimum device output conductance of $g_{o}$, and a minimum capacitance level of $C_{T}$. In this respect a device may be either a single transistor or a compound device such as a cascode transistor. We also assume that the opamp dominant pole is determined by the minimum capacitance level $C_{T}$ in conjunction with the minimum device output conductance $g_{o}$, i.e. $\omega_{0}=g_{o} / C_{T}$. This is the situation which can be achieved with a single high impedance node forming the dominant pole. All other nodes are assumed to have an impedance level of $\left(1 / g_{m}\right) \|\left(1 / s C_{T}\right)$. In particular, we must require that the impedance level of the feedback network does not introduce high impedance nodes. Also, low frequency voltage or current gains cannot be higher than $g_{m} / g_{0}$.

\begin{tabular}{lcccc}
\hline $\begin{array}{l}\text { Opamp } \\
\text { type }\end{array}$ & $\begin{array}{c}\text { Open loop } \\
\text { gain, } A_{0}\end{array}$ & $\begin{array}{c}\text { Feedback } \\
\text { resistors }\end{array}$ & $R_{L}$ & $R_{S}$ \\
\hline VCVS & $g_{m} / g_{\circ}$ & $R_{2}<1 / g_{m}$ & $>1 / g_{m}$ & $<1 / g_{o}$ \\
CCCS & $g_{m} / g_{o}$ & $R_{2}<1 / g_{m}$ & $<1 / g_{o}$ & $>1 / g_{m}$ \\
CCVS & $1 / g_{\circ}$ & - & $>1 / g_{m}$ & $>1 / g_{m}$ \\
VCCS & $g_{m}\left(g_{m} / g_{o}\right)$ & $\begin{array}{c}R_{1}<1 / g_{m} \\
\text { and } \\
R_{2}<1 / g_{m}\end{array}$ & $\simeq 1 / g_{m}{ }^{(a)}$ & $<1 / g_{\circ}$ \\
\hline
\end{tabular}

(a) Only for the I-V converter. Otherwise $R_{L}<1 / g_{0}$.

Table 4. Technology constraints in integrated feedback amplifiers.
The implications of these technology constraints are summarized for each of the opamp types in Table 4. Using the opamp and isolation buffer parameters of Table 4 together with the feedback network impedance levels given in Table 4 we can compute the set of technology limited maximum low frequency loop gains given in Table 5 . The closed loop bandwidth is found by multiplying the low frequency loop gain by $\omega_{o} /(2 \pi)=g_{o} /\left(2 \pi C_{T}\right)$. This table reveals that none of the constant-bandwidth configurations have a loop gain potential exceeding the loop gain potential of the constant gain-bandwidth product configurations. Thus, it also discloses the (perhaps controversial) fact that the maximum loop gain potential is found in the configurations exhibiting a constant gain-bandwidth product relation, $G_{0} \times B W=A_{0} \omega_{0} /(2 \pi)$. Further, it shows that full exploitation of the loop gain and bandwidth potential in the so-called constant-bandwidth configurations results in loop gain and bandwidth relations which are strongly dependent on the closed loop gain.

These results have been reported previously in the case of the transimpedance opamp used for a voltage to voltage converter [3] but to the knowledge of this author it has not been reported for the other configurations. With these observations in mind one might be tempted to conclude that only the constant gain-bandwidth product configurations in Table 5 are of interest in high speed design. However, this is taking the conclusion too far. What is not apparent from the table is that with $C_{T}$ selected as the minimum capacitance which the technology can provide, the simple opamp model with a single dominant pole holds true only for high values of closed loop gain. For lower values of closed loop gain the compensation capacitor must be increased in order ensure stability. In practice, the compensation capacitor is often an internal, fixed capacitance and its value is set to ensure stability under all normal op- 


\begin{tabular}{lcccc}
\hline $\begin{array}{l}\text { Opamp } \\
\text { type }\end{array}$ & $\begin{array}{c}\text { V-V } \\
\text { converter }\end{array}$ & $\begin{array}{c}\text { I-I } \\
\text { converter }\end{array}$ & $\begin{array}{c}\text { I-V } \\
\text { converter }\end{array}$ & $\begin{array}{c}\text { V-I } \\
\text { converter }\end{array}$ \\
\hline VCVS & $\frac{1}{G_{0}} \frac{g_{m}}{g_{o}}$ & $\frac{1}{G_{0}+\frac{g_{o}}{g_{m}}\left(G_{o}+1\right)} \frac{g_{m}}{g_{o}}$ & $\frac{g_{m}}{1+R_{2} g_{o}} \frac{1}{g_{o}}<\frac{1}{G_{0} g_{o}}$ (a) & $\frac{1}{G_{0}+g_{m}} g_{m} \frac{g_{m}}{g_{o}}$ \\
\hline CCCS & $\frac{1}{G_{0}+\frac{g_{o}}{g_{m}}\left(G_{o}+1\right)} \frac{g_{m}}{g_{o}}$ & $\frac{1}{G_{0}} \frac{g_{m}}{g_{o}}$ & $\frac{g_{m}}{1+R_{2} g_{o}} \frac{1}{g_{o}}<\frac{1}{G_{0} g_{o}}$ (a) & $\frac{1}{G_{0}+g_{m}} g_{m} \frac{g_{m}}{g_{o}}$ \\
\hline CCVS & $\frac{1}{G_{0}+R_{2} g_{m}} \frac{g_{m}}{g_{o}}$ & $\frac{1}{G_{0}+R_{2} g_{m}} \frac{g_{m}}{g_{o}}$ & $\frac{1}{R_{2} g_{o}}=\frac{1}{G_{0} g_{o}}$ & $\frac{1}{G_{0}+2 g_{m}} g_{m} \frac{g_{m}}{g_{o}}$ \\
\hline VCCS & $\frac{1}{G_{0}+\frac{g_{o}}{g_{m}} G_{o}} \frac{g_{m}}{g_{o}}$ & $\frac{1}{G_{0}+\frac{g_{o}}{g_{m}} G_{o}} \frac{g_{m}}{g_{o}}$ & $\frac{g_{m}}{1+R_{2} g_{o}+\frac{g_{o}}{g_{m}}} \frac{1}{g_{o}}<\frac{1}{G_{0} g_{o}}$ (a) & $\frac{1}{G_{0}} g_{m} \frac{g_{m}}{g_{o}}$ \\
\hline
\end{tabular}

(a) Note that $g_{m}<1 / R_{2}=1 / G_{0}$

Table 5. Loop gain potential of feedback amplifiers.

erating conditions, thus essentially wasting the bandwidth potential. In constant-bandwidth configurations, stability can be controlled by the feedback resistors, simplifying the optimization of the device. Another important issue not addressed here is the large signal bandwidth. In many opamp implementations the compensation capacitor leads to a slew rate limitation, causing the large signal bandwidth to be smaller than the small signal bandwidth. This limitation is avoided if class $A B$ stages are used to drive the compensation capacitor. This configuration is normal practice in the case of transresistance opamps (current feedback opamps) but it is unusual (though not impossible) in the case of voltage opamps [3].

\section{CONCLUSION}

We have analyzed feedback amplifiers realizing all of the four basic converters, i.e. voltage-to-voltage, current-tocurrent, current-to-voltage, and voltage-to-current. When implemented with non-ideal opamps these configuration have a constant product of closed loop gain and closed loop bandwidth if the opamp is of the same type as the feedback converter, i.e. voltage opamp, current opamp, transresistance opamp, and transconductance opamp. If the opamp is of a different type than the feedback converter, a bandwidth which is independent of the closed loop gain is found, provided the load and source impedances can be isolated from the opamp through the use of ideal voltage or current buffers. The results even indicate that with ideal buffers we have the captivating situation that the closed loop bandwidth may equal the unity gain bandwidth of the opamp regardless of the closed loop gain.

Next, we have introduced some generally applicable assumptions concerning the properties of the opamps, the impedance levels of the feedback resistors, and the impedance levels achievable from the input and output isolation buffers. With the limitations arising from these technology assumptions we have calculated the potential for loop gain and closed loop bandwidth for each of the feedback converters. This calculation brings forth the conclusion that the constant gain-bandwidth product configurations provide the highest potential loop gain and the highest closed loop bandwidth.

The conclusion above does not take into account ease of implementation, limitations such as large signal bandwidth, or more complex frequency response characteristics of the opamps involved. Thus, in a practical design one may find a more complex scenario than outlined in this paper. However, it is believed that the results demonstrated in this paper are important as guidelines in the selection of design approaches in high frequency feedback systems.

\section{REFERENCES}

[1] C. Toumazou, F. J. Lidgey, and D. G. Haigh, Eds., Analogue IC design: the current mode approach. London, UK: Peter Peregrinus Ltd., 1990, pp. 127-180.

[2] C. Toumazou, A. Payne, and J. Lidgey, "Current-Feedback Versus Voltage Feedback Amplifiers: History, Insight and Relationships," Proc. 1993 IEEE International Symposium on Circuits and Systems, (ISCAS'93), pp. 1046-1049.

[3] E. Bruun, "Feedback Analysis of Transimpedance Operational Amplifier Circuits," IEEE Trans, Circuits and Systems-I, vol. 40, pp. 275-278, 1993. 